TITLE:

\title{
Viscoelastic fluid behaviors around a rising bubble via a new method of mesh deformation tracking
}

\author{
$\operatorname{AUTHOR}(\mathrm{S}):$ \\ Imaizumi, Yuya; Kunugi, Tomoaki; Yokomine, \\ Takehiko; Kawara, Zensaku
}

\section{CITATION:}

Imaizumi, Yuya ... [et al]. Viscoelastic fluid behaviors around a rising bubble via a new method of mesh deformation tracking. Chemical Engineering Science 2014, 120: 167-173

\section{ISSUE DATE:}

2014-12

URL:

http://hdl.handle.net/2433/192776

\section{RIGHT:}

(c) 2014 Elsevier Ltd.; この論文は出版社版でありません。引用の際には 出版社版をご確認ご利用ください。; This is not the published version. Please cite only the published version. 


\title{
Viscoelastic fluid behaviors around a rising bubble via a new method of mesh deformation tracking
}

\author{
Yuya Imaizumi*, Tomoaki Kunugi, Takehiko Yokomine, and Zensaku Kawara \\ Department of Nuclear Engineering, Kyoto University, Kyoto-Daigaku Katsura, Nishikyo, Kyoto, 615-8540, Japan
}

\begin{abstract}
The deformation of a hydrogen microbubble line and/or mesh in a viscoelastic fluid around a rising bubble was tracked from the original static position in order to discuss the mechanism of the typical phenomena such as the negative wake or the cusp shape. This new experimental method is essentially important because of the hysteresis-dependent nature of the viscoelastic fluid. This new method makes this study distinctive from a number of conventional studies of viscoelastic fluids focusing on the non-Newtonian properties and/or the instantaneous flow field. According to our experimental results, the flow mechanism responsible for the negative wake or cusp shape was attributed to the accumulation and release of the shear strain energy. Some residual displacements were observed after the bubble rising, which were almost completely reproduced as the internal dissipations in a Maxwell model modified with a non-linear spring.
\end{abstract}

Keywords: Viscoelastic fluid, Rising bubble, Tracking, Negative wake

\section{Introduction}

Behaviors of viscoelastic fluids of polymer solutions play important roles in chemical engineering. They can also be applied as functional fluids distinguished by not only the effect of significant drag reduction on the turbulent flows called "Toms effect" (Toms, 1949), but also the temporary accumulation and release of the strain energy. However, there seems to be few studies related the viscoelastic multiphase flow. Therefore, as a starting point for the research of the viscoelastic gas-liquid two-phase flow, a viscoelastic fluid behavior around a rising bubble was studied.

It is well known that there are typical phenomena such as a "negative wake" whose flow direction is opposite from the usual wake and a cusp shape forms at the bottom of a rising bubble in a non-Newtonian fluid (Astarita, 1965; Hassager, 1979). Non-Newtonian properties such as a shearthinning are common in polymeric liquids which are also known to have viscoelasticities. Other than the earlier studies (Bisgaard and Hassager, 1982; Maalouf and Sigli, 1984; Arigo and McKinley, 1998) that measure flow speed at a fixed point using a laser-doppler anemometer (LDA), instantaneous global flow fields around a rising bubble (Funfschilling and Li, 2001; Kemiha et al., 2006; Lin and Lin, 2005) have recently been obtained by using a particle image velocimetry (PIV) technique.

\footnotetext{
*Corresponding author. Tel.: +81 902921 5001; fax: +81 75383 3924

Email address: imaizumiyuuya@nucleng.kyoto-u.ac.jp (Yuya Imaizumi)
}

However, the origin of the negative wake is still unclear (Frank and Li, 2006) and furthermore, some contradicting arguments (Harlen, 2002) exist. There are some reports in which the negative wake was observed (Maalouf and Sigli, 1984) only in the shear-thinning fluid but not in the Bogar fluid, a fluid with an elasticity and a constant viscosity. In addition, in Bogar fluids, an "extended wake" (Arigo et al., 1995) was observed rather than the negative wake. However, the negative wake in the Bogar fluid was reproduced by a numerical simulation using the FENE-CR model (Satrape and Crochet, 1994). Moreover, the simulations of fluids with the shear-thinning and without the viscoelasticity (Zhang et al., 2010; Ohta et al., 2003) do not reproduce any cusp shapes or negative wakes. This discrepancy might be due to the difficulty of experimentally discriminating the contributions of the shear-thinning and the viscoelasticity. Since the deformed bubble shape or the gas-liquid interface phenomenon affects the flow pattern, the wake and the shape of the rising bubble would be affected by them. However, the negative wake has been observed experimentally even around a settling sphere (Arigo and McKinley, 1998; Kemiha et al., 2006). Thus, the mechanism of negative wake would be caused by the viscoelasticity especially for its elasticity, and further experimental and/or theoretical investigations would be required as previously pointed out (Funfschilling and Li, 2001).

Moreover, viscosities that were obtained by viscometers cannot be applied to the temporal and unsteady motions of viscoelastic fluids such as the single bubble rising. In general, viscometers only deal with the ultimate steady shear flows in order to obtain non-Newtonian properties which are the unproportional dependencies of shear 
stresses on the various shear speeds. However, the shear stresses of viscoelastic fluids depend on the shear strains due to their elasticities which polymer solutions of nonNewtonian fluids would have inevitably.

In addition to the elasticities, there are the hysteresis effects in viscoelastic fluids as follows. As is well known, the viscosity is a property of stress due to an instantaneous shear speed, while the elasticity is a property of stress caused by a stored strain. The stress in an inelastic viscous fluid thus depends only on the instantaneous shear speed; the shear stress in an elastic body depends on the stored shear strain from the original state. As for viscoelastic fluids which have both properties, the strain is accumulated in the fluid under the shear and it gradually relaxes. Therefore, the stress depends on the latent strain. Viscoelastic fluids have these hysteresis effects due to the previously loaded shear unlike viscous fluids. Thus, the instantaneous flow field obtained by the PIV would be inadequate to discuss the mechanism of viscoelastic fluids.

Therefore, in this study, we tracked all the deformations of viscoelastic fluid elements from the original static state using the hydrogen microbubble line and/or mesh. This new method, called "Mesh Deformation Tracking", enables us to evaluate the viscoelastic flow characteristics quantitatively and deterministically. Flow mechanisms regarding the negative wake and/or the cusp shape have been considered and provided consistency with our experimental results.

\section{Experimental setup}

SAP (Sodium Acrylate Polymer) solution, known to have a strong elastic effect and obvious shear-thinning (Ohta et al., 2003), was used as a working fluid. The concentration was set to $0.3 \%$ where the rising speed of bubble and the typical behaviors were suitable to be observed. As a comparative experiment regarding an inelastic viscous fluid, the aqueous sugar solution of $60 \%$, which has a relatively high viscosity, was used.

Experimental setup is shown in Fig. 1. The rectangular bubble column was made of acrylic plates that were 10 $\mathrm{mm}$ thick and the diameters were $130 \mathrm{~mm}$ in width, 890 $\mathrm{mm}$ in height of its inner diameters, and $810 \mathrm{~mm}$ in fluid depth. The volume of air quantified by the syringe was injected through a needle and accumulated in the hemisphere installed at the bottom of the bubble column. The bubbles started to rise when the hemisphere was rotated by drawing the control strings. The range of the bubble volume was from 0.5 to $50 \mathrm{ml}$. The relatively large bubbles having the maximum horizontal diameters of about $70 \mathrm{~mm}$ were used to observe the phenomena. Lycopodium powder, average diameter of $30-40 \mu \mathrm{m}$, was suspended in the fluid as tracers for the PIV. They were illuminated with a light sheet $4 \mathrm{~mm}$ thick by a lighting system consisted of a broad light source, a cylindrical lens, and a slit. The light intensity was amplified by a mirror attached at the opposite side of the column.
In the fluid, the hydrogen microbubbles are nearly stationary, and either a line or a two-dimensional mesh of microbubbles was tracked to visualize the fluid motion. Hydrogen microbubbles were generated by the electrolysis of water by direct voltage of $24 \mathrm{~V}$ in the line tracking and $194 \mathrm{~V}$ in the mesh tracking. For the negative electrode, a horizontal platinum wire was used in the line tracking a platinum mesh was used in the mesh tracking. The wire diameter was $100 \mu \mathrm{m}$ for the line tracking and $50 \mu \mathrm{m}$ for the mesh tracking with mesh intervals of $7.5 \mathrm{~mm}$ in all directions. For the positive electrode, a platinum wire of 100 $\mu \mathrm{m}$ was used in the line tracking and 11 platinum wires with intervals of $10 \mathrm{~mm}$ were used in the mesh tracking. The conduction time of electrical current was set to about $0.2 \mathrm{sec}$ in each case and the electrical current was 182 $\mathrm{mA}$ in the line tracking and $3.17 \mathrm{~A}$ in the mesh tracking. The copper frame of the mesh was electrically insulated by painting with ink. After the microbubble generation, the wire or the mesh was cut or removed to prevent the splitting of the rising bubble. The fluid temperature was kept at $12{ }^{\circ} \mathrm{C}$ for the PIV and the line tracking and at $16{ }^{\circ} \mathrm{C}$ for the mesh tracking. Since the hydrogen bubble method has often been used to visualize a timeline in a flow channel, it can be adopted in this experiment to track the flow motion that occurred in an initially static fluid.

Fluid motions were recorded by a high speed camera (Photoron FASTCAM 512PCI) with resolution of $512 \times 512$ pixels, with camera speeds of $125 \mathrm{fps}$ and $250 \mathrm{fps}$, the exposure times of $8 \mathrm{~ms}$ and $4 \mathrm{~ms}$, for the PIV and the line tracking and for the mesh tracking, respectively. The images were analyzed by the software, OpenPIV, and the flow fields were visualized by MicroAVS.

\section{Experimental results}

\subsection{Bubble shapes, and flow fields by PIV}

The cusp shapes were observed in all the bubble volumes from 0.5 to $50 \mathrm{ml}$. The negative wake was observed under the conical upward flow zone behind the bubble in this fluid as well as the previous studies for PIV (Funfschilling and Li, 2001; Kemiha et al., 2006) in which different test fluid was used. This result was taken into account qualitatively in the section 5 in order to discuss the viscoelastic flow mechanism. On the other hand, no negative wakes and/or cusp shapes were observed in the flow fields of the sugar solution for comparative experiments with the same bubble volumes.

\subsection{Relationship between the shape change of the rising bubble and the flow speed of the negative wake}

For bubble volumes of 4.0-8.0 ml, the bubble changed its shape while rising. Furthermore, when the bubble was stretched vertically, the negative wake speed slowed. Figs 2 and 3 show different bubbles with the same volumes of $7.0 \mathrm{ml}$ and Fig 4 shows the relationship between the bubble shape and the negative wake speed. In the flow 


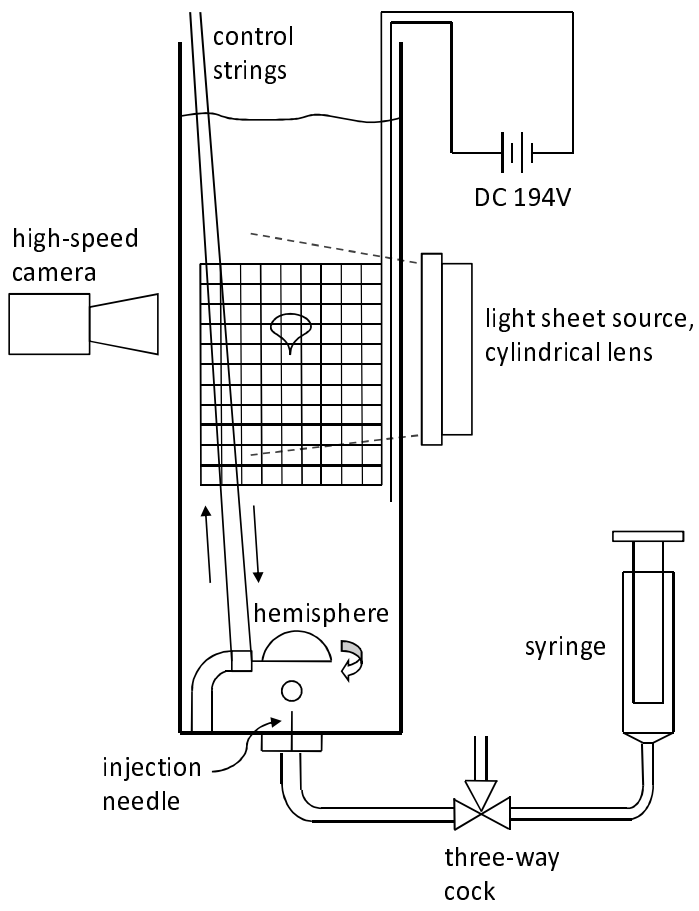

Figure 1: Setup for the mesh tracking experiment. Microbubbles were generated by electrolysis and tracked during the rising of a bubble.

field around the bubble in Fig. 3, the conical upward flow and the negative wake became significantly weak compared to those of the bubble with a short vertical length.

\subsection{Tracking of microbubble line}

The experimental results for tracking the microbubble lines are shown in Fig. 5 for the case of a $25 \mathrm{ml}$ bubble. The elapsed times from the moment when the top of the bubble reached the original position of the line are described below the figures. The line was entrained upward, distorted especially at its horizontal center (Fig. 5(b)(c)), and almost returned downward (Fig. 5(d)-(e)) to its original position. However, a certain amount of residual displacement is observed in the ultimate state (Fig. 5(f)),
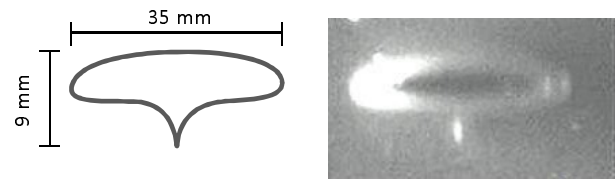

Figure 2: Bubble shape in $7.0 \mathrm{ml}(1)$
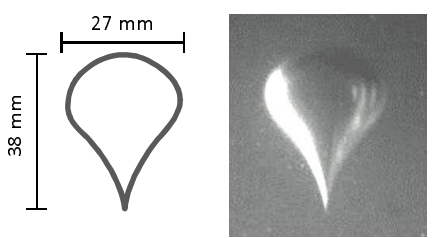

Figure 3: Bubble shape in $7.0 \mathrm{ml}(2)$

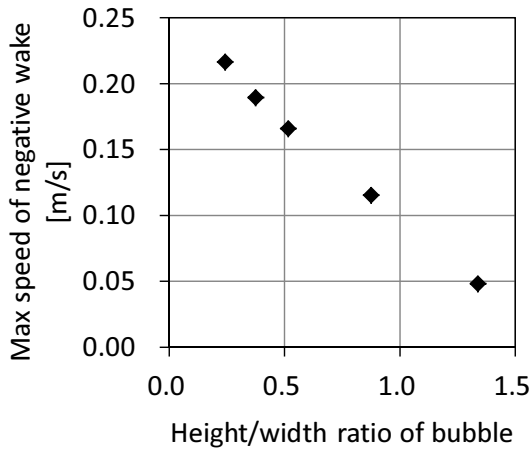

Figure 4: Relationship between the cusp elongation and the speed of the negative wake for the same bubble volumes of $7.0 \mathrm{ml}$

which remained as long as observed. Fig. 6(a)-(h) shows the case of a $15 \mathrm{ml}$ bubble. The residual displacement was small compared to that in the case of the $25 \mathrm{ml}$ bubble. There were hardly any residual displacements for a bubble of less than $8 \mathrm{ml}$.

In the case where the platinum wire was not cut, the rising bubble was split by the wire, which is attributed to the well-known filament stability (Bird et al., 1987) of the polymeric liquids. An example of a $25 \mathrm{ml}$ bubble is shown in Fig. 7. In this split case, the residual displacement was significantly smaller compared to that in the previous case of a non-splitting bubble. This can be attributed to the smaller strain in the fluid.

\subsection{Tracking of mesh deformation}

The microbubble mesh tracking is composed of the line-tracking for both lateral and longitudinal microbubble lines. Fig. 8 shows the process of deformation of a microbubble mesh for a $15 \mathrm{ml}$ bubble. The elapsed times from the moment when the top of the bubble reached the frame bottom are described. In general, deformation of each mesh element corresponds to the fluid strain at the position. Unlike the microbubble line tracking in which we could not track the fluid movement along the line due to the undistinguishable microbubble motion, all directions of the movements can be tracked by this microbubble mesh deformation tracking. The large deformation was observed especially in the vicinity of the rising bubble. While the movements of lateral lines were large and took time to return to the original positions, the longitudinal lines returned immediately. Furthermore, the lateral lines had some residual displacements and the longitudinal ones had little. This generally indicates that the motions of the viscoelastic fluids are mainly along a moving obstacle, and the motion perpendicular to it immediately returns and leaves almost no displacements.

\section{Evaluation of internal dissipation}

\subsection{Calculation procedure}

The residual displacements for the microbubble mesh tracking are due to the internal dissipations of the vis- 


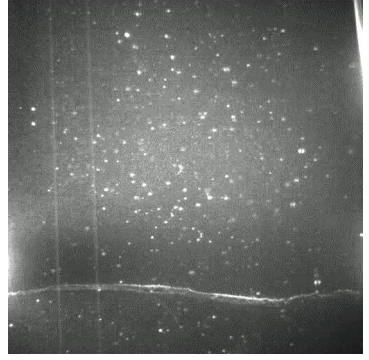

(a) $-0.440 \mathrm{sec}$

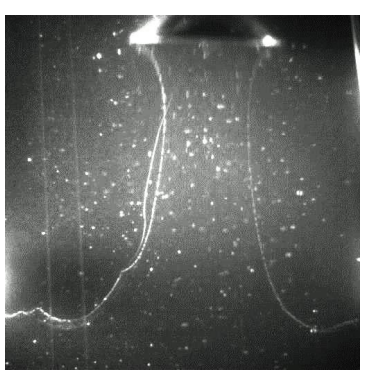

(c) $0.296 \mathrm{sec}$

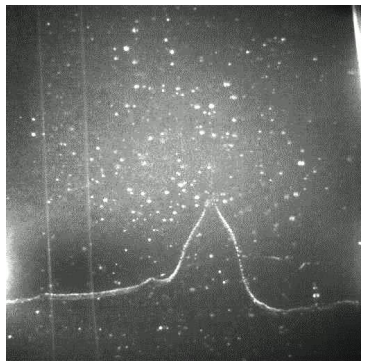

(e) $3.448 \mathrm{sec}$

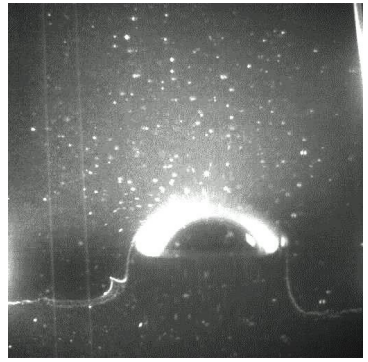

(b) $0.088 \mathrm{sec}$

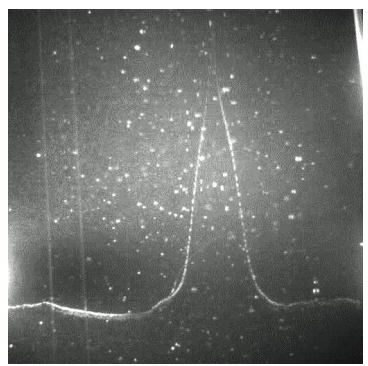

(d) $1.160 \mathrm{sec}$

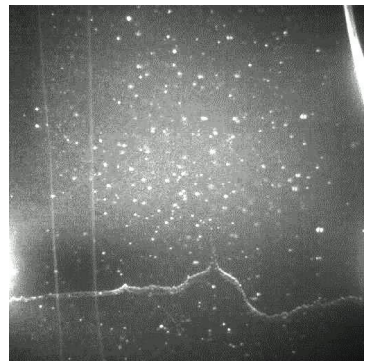

(f) $4 \mathrm{~min}$
Figure 5: Motion of a microbubble line for a $25 \mathrm{ml}$ bubble. The elapsed times from the moment when the top of the bubble reached the original position of the line are described below the figures. There was a residual displacement in the ultimate state (f) compared to the original static state (a).

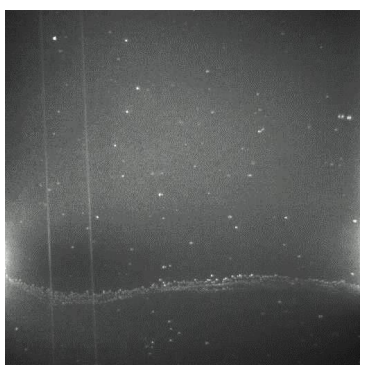

(a) $-0.600 \mathrm{sec}$

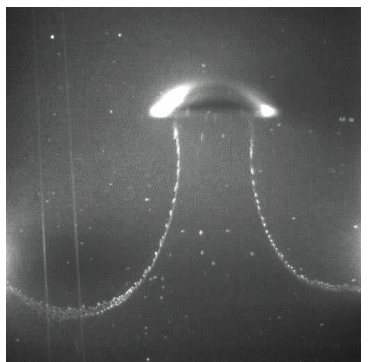

(c) $0.208 \mathrm{sec}$

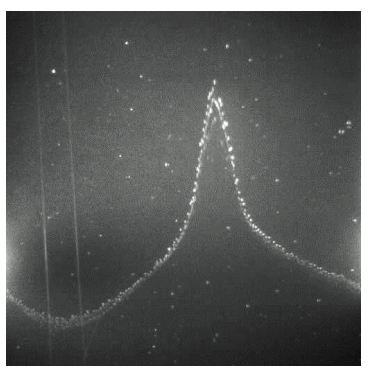

(e) $0.744 \mathrm{sec}$

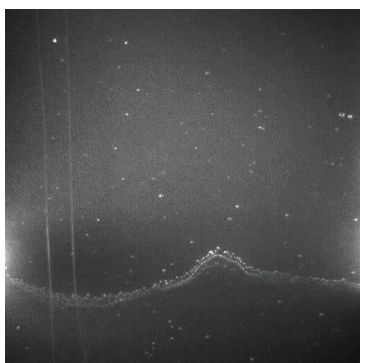

(g) $6.352 \mathrm{sec}$

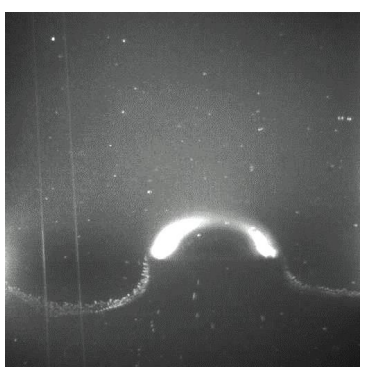

(b) $0.072 \mathrm{sec}$

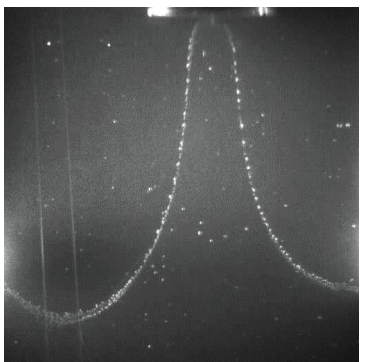

(d) $0.312 \mathrm{sec}$

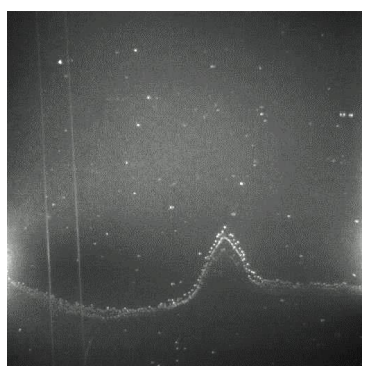

(f) $1.168 \mathrm{sec}$

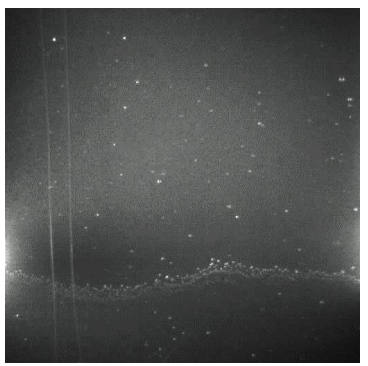

(h) $3 \mathrm{~min}$
Figure 6: Motion of a microbubble line for a $15 \mathrm{ml}$ bubble. 


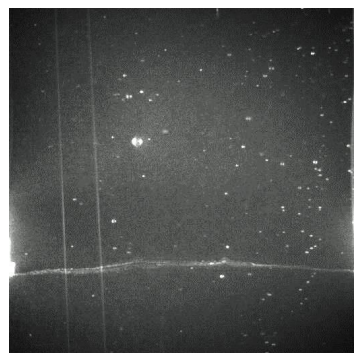

(a) $-0.376 \mathrm{sec}$

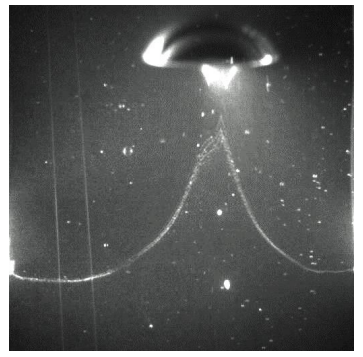

(c) $0.248 \mathrm{sec}$

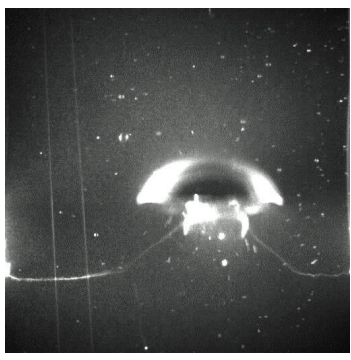

(b) $0.104 \mathrm{sec}$

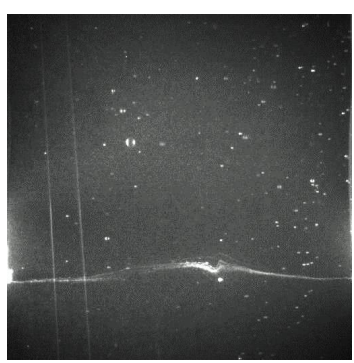

(d) $1.824 \mathrm{sec}$
Figure 7: Motion of a microbubble line for a $25 \mathrm{ml}$ bubble. In this case, the bubble was split by the horizontal wire.

coelastic fluid. The internal dissipation in a viscoelastic model were calculated and compared with the experimental result. Line segments including the viscoelastic model were configured in the calculation domain as well as the experimental mesh. The internal dissipation calculation was integrated by the time increment which corresponds to the experimental images. This means that the internal dissipation was calculated from all the experimental images from the initial static moment to the final moment of calculation stop. The shapes of the lines after the inertial motions settled down were compared from the calculation with the experimental results to assess the validity of this calculation. In this calculation, the value was updated by referring to the experimental image each time, which can be called a one-way method.

Fig. 9 is the Maxwell model element implemented in the line segments of the calculation domain. To correspond with the experimental result, a non-linearity of the relationship between the force and the displacement was implemented in the spring of the Maxwell model as follows:

$$
|\sigma|=k\left|\gamma_{e}\right|^{n}, \quad \operatorname{sgn} \sigma=\operatorname{sgn} \gamma_{e}
$$

Then, time variation of the dissipation $\Delta \gamma_{d}$ can be obtained by,

$$
\Delta \gamma_{d}=\operatorname{sgn}\left(\gamma-\gamma_{d}\right) \cdot \Delta t\left|\gamma-\gamma_{d}\right|^{n} / \lambda
$$

where denotations are $\sigma$ : force, $\gamma$ : total displacement, $n$ : degree of non-linearity of the spring, $\gamma_{e}$ : displacement in spring, $\gamma_{d}$ : displacement in dashpot, $\eta$ : viscosity coefficient, $k$ : elasticity coefficient, $\lambda(=\eta / k)$ : relaxation parameter. The values shown without units are all in SI.

The $\gamma$ was obtained by,

$$
\gamma=L-L_{0}
$$

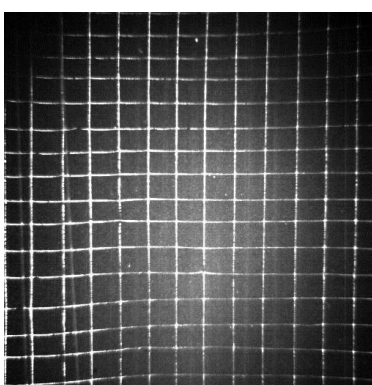

(a) $-0.452 \mathrm{sec}$

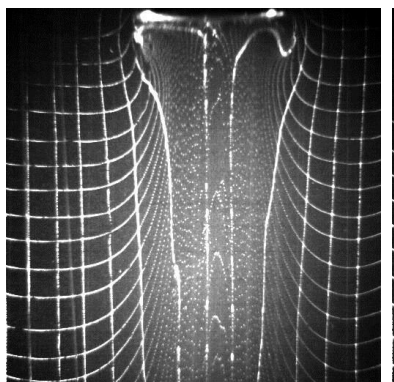

(c) $0.348 \mathrm{sec}$

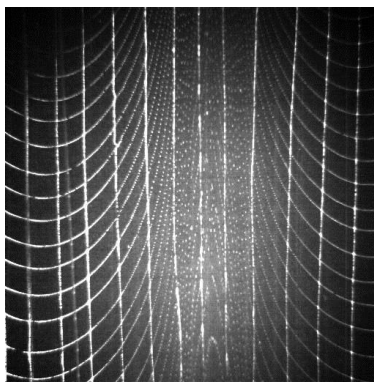

(e) $0.848 \mathrm{sec}$

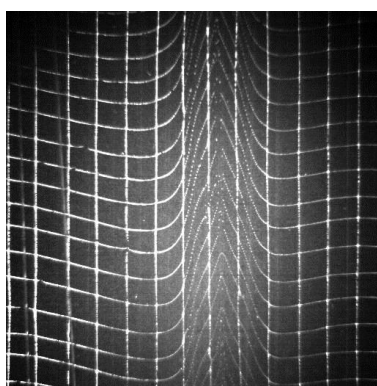

(g) $1.680 \mathrm{sec}$

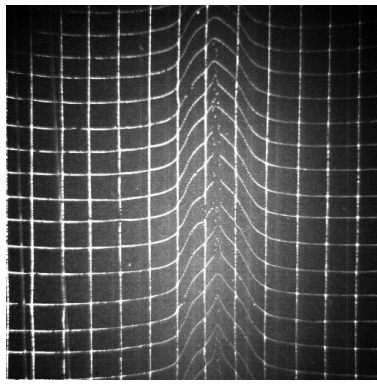

(i) $6.416 \mathrm{sec}$

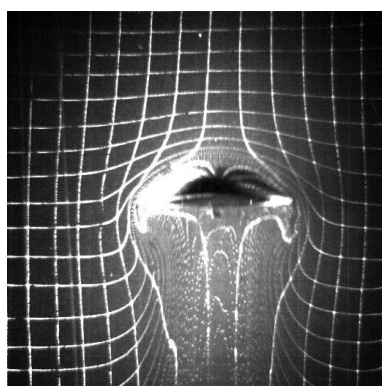

(b) $0.184 \mathrm{sec}$

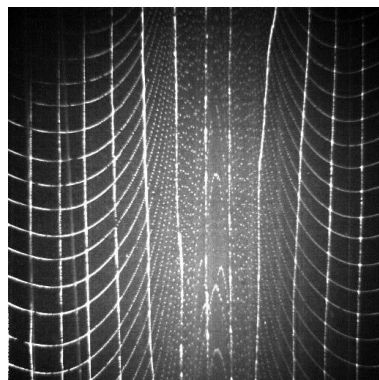

(d) $0.572 \mathrm{sec}$

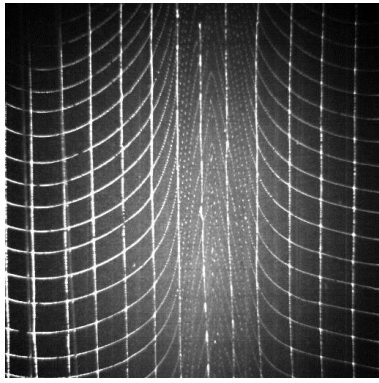

(f) $1.236 \mathrm{sec}$

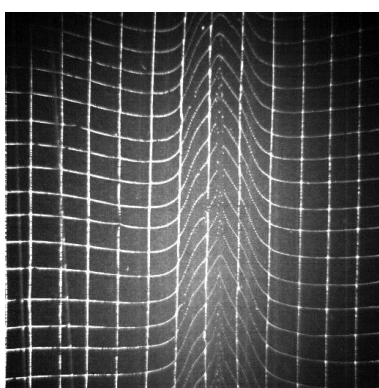

(h) $2.208 \mathrm{sec}$

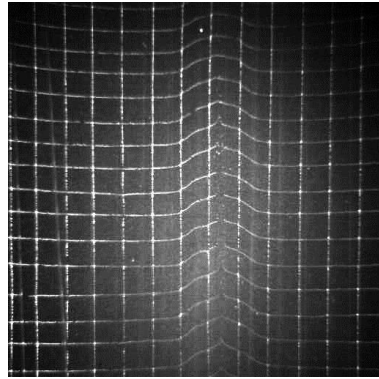

(j) $4 \mathrm{~min}$
Figure 8: Mesh deformation of microbubbles for a $15 \mathrm{ml}$ bubble. The elapsed times from the moment when the top of the bubble reached the frame bottom are described. There were residual displacements in the ultimate state $(\mathrm{j})$ compared to the original static state (a). 


\section{$-\mathrm{Wh}-\mathrm{T}$}

Figure 9: Maxwell model consisted of spring and dashpot

where $L$ is a distance between the mesh points obtained each time from the experimental images of mesh tracking and $L_{0}$ is its initial value.

The length of the line segments protruding from the observation frame was obtained by the following method. Assuming that the fluid motions are always equal from a view point of the rising bubble, the time-shifting deformation, where the time-shift value is the time for the bubble to pass through the frame, is observed at the frame above or below the present observed frame. A long composite image was obtained by the vertical disposition. The elapsed time, $t$, is defined downward in this composite image and interpolated for each horizontal line. The time interval, $\Delta t$, for the calculation is divided into $22 \mathrm{~ms}$, corresponding to the initial mesh interval.

For three-dimensionality, a rotor with an axis corresponding to the bubble center was considered. Even then, the relaxation time, $\lambda$, is independent of the radius, $r$, because both of $\eta$ and $k$ are proportional to $r$ due to the proportionality of the circumferential length of an annulus ring sheet which is a part of the rotor.

\subsection{Calculation results}

A lateral line of the mesh was described by converting $\gamma_{d}$ to the vertical displacements $z$. Fig. 10 shows the shapes of the lines of the experiment and calculation. The agreement is quite good when $n$ and $\lambda$ are tuned to the optimal values. In the case of the linear spring $(n=1)$, the lines cannot be fit however $\lambda$ is tuned.

Fig. 11 shows the time variation of $\gamma$ obtained by the experiment and $\gamma_{d}$ by the calculation. The $\gamma$ approaches $\gamma_{d}$ when $\lambda$ is tuned to the optimal value. The $\gamma$ does not follow an ideal asymptotical line because of the contraction and rebounding from the inertia and elasticity.

\section{Discussions on viscoelastic mechanism around a rising bubble}

In our experiment, the downward flow toward the original static position was observed after the bubble rising. This downward flow to recover the strain corresponds to the negative wake whose mechanism has been debated for a long time. According to the experimental results, the mechanism of the negative wake can be considered as follows. As illustrated in Fig. 12, the shear in the conical upward flow induced by the rising bubble is accumulated as the strain in the fluid. This strain produces the stress to release the elastic energy, which drives the fluid downward to form the negative wake. In a similar way, the formation of the cusp shape can be explained by the aforementioned stress that it draws the fluid just below the bubble.

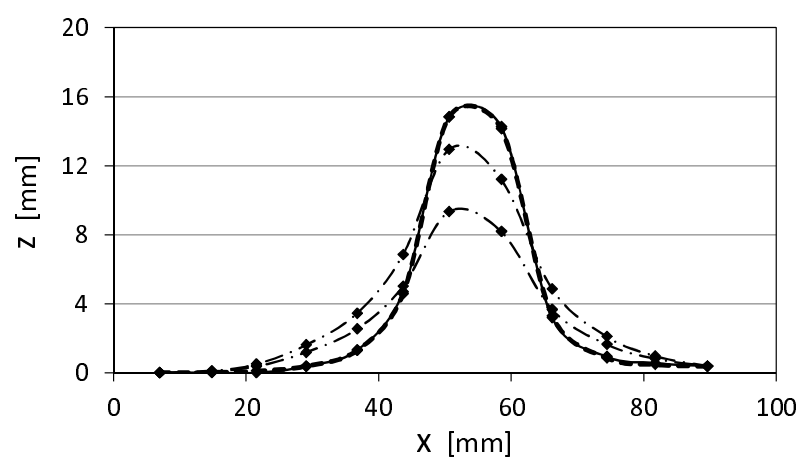

Figure 10: Lines of microbubbles from the experimental image (solid line) and those of the calculation (dashed line, dashed-dotted lines). The dashed line where the parameters are tuned to the optimal values ( $n=2.39, \lambda=0.19$ ) is almost overlapping with the experimental one. The two dashed-dotted lines are the values of the linear spring $(n=1)$, where $\lambda=36$ in the upper line and $\lambda=70$ in the lower line. The elapsed time, $t$, of these lines is $5.56 \mathrm{~s}$ and is explained in Fig. 11 .

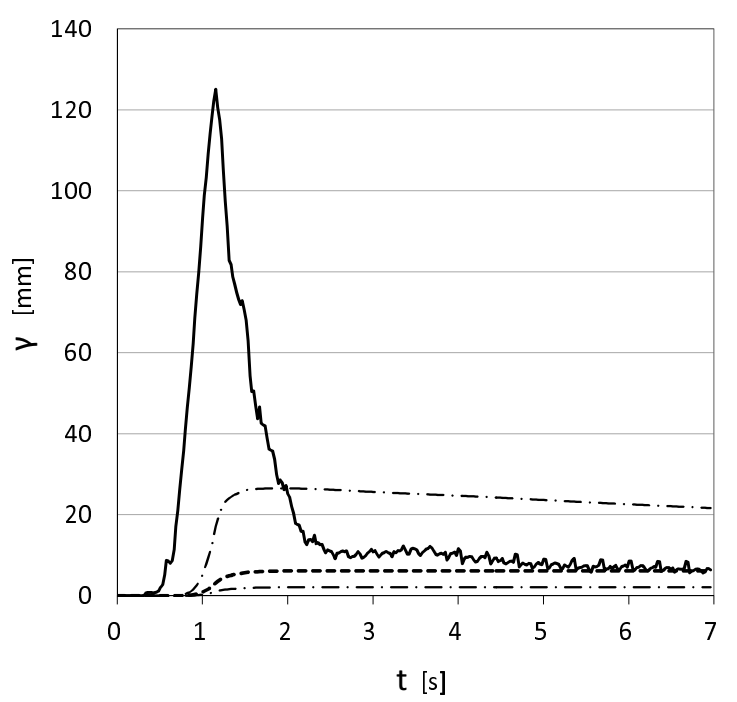

Figure 11: Time variation of the experimental displacement $\gamma$ (solid line) and the calculated internal dissipations $\gamma_{d}$ (dashed line, dasheddotted lines). The $\gamma$ approaches $\gamma_{d}$ asymptotically only when $\lambda=$ 0.19 (dashed line). The two dashed-dotted lines are the $\gamma_{d}$ when $\lambda=0.06$ (upper) and $\lambda=0.6$ (lower). The $n$ is 2.39 as shown in Fig. 10. The start point of the elapsed time, $t$, is fixed to $0.445 \mathrm{~s}$ before the deformation start of the line. The $\gamma$ or $\gamma_{d}$ of the vertical coordinate is a sum of the 5 th and 6 th lateral line segments from the left in Fig. 10. 


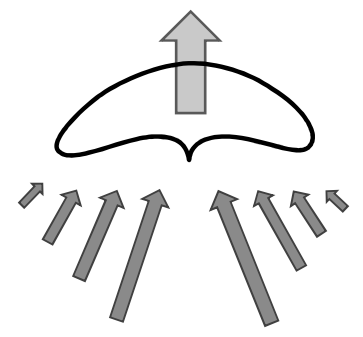

(a) Accumulation of the elastic energy of shear strain

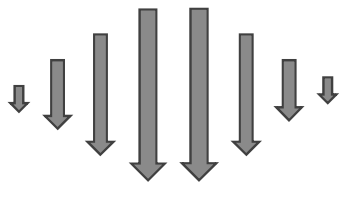

(b) Release of the elastic energy of shear strain
Figure 12: Illustration for the mechanism of the generation of the negative wake and cusp shape. (b) is the succeeding stage after the stage of (a).

This mechanism also gives an explanation for our observed phenomena shown in Fig. 4. The negative wake became weak in the case of the elongated bubble. Because of the elongated bubble shape, the flow speed and the shear in the upward flow became weak, which provided the small stored energy and eventually produced the small driving force for the negative wake.

The vertical displacement of the lateral line after the convergence of inertial motion was almost coincident with that obtained by the calculation based on the Maxwell model modified with the power-law spring. The non-linear spring, which stiffens when it is extended, has been implemented in the conventional dumbbell-models, such as the FENE model (Bird et al., 1987). This assumption can be attributed to the mechanism of stretching molecules with finite length that are entangled and shrunk initially. The linear polymers that were initially shrunk cannot extend moreover when they are stretched and aligned in the same direction. The elastic force occurs in order to increase the entropy by getting away from the aligned composition with low entropy, which is known as the molecular mechanism of the rubber elasticity. The molecular displacement occurs by this internal elastic force to relax it, which corresponds to the dissipation or force relaxation macroscopically. This would be the physical reasonability of this Maxwell model modified with the non-linear spring.

\section{Conclusions}

The mechanism of viscoelastic fluids cannot only be caused by the instantaneous flow field obtained by the PIV because of the hysteresis dependencies of the viscoelastic fluids. Therefore, the tracking of the deformation is essential and indispensable for the discussion of the elastic behavior of the viscoelastic fluid. In this study, all the deformations of the viscoelastic fluid were tracked from the original static position by the electrically generated hydrogen microbubble line or mesh to investigate the characteristic behaviors of the viscoelastic fluid, such as the negative wake or the cusp shape whose mechanisms were unclear for decades.
One of the significance of this mesh deformation tracking is to track whether the fluid returns to the original static position or not. In our experimental results, it was observed that the induced deformations by the rising bubble are always almost restored to the original static position. This experimental result suggests the long-debated origin of the negative wake and/or the cusp shape was caused by releasing the accumulated elastic energy of the shear strain in the viscoelastic fluid. This mechanism also gives an explanation for our observed phenomenon that the negative wake became weak in the case of the elongated cusp.

In the ultimate position of the microbubble mesh or line tracking, there were residual displacements. The residual displacements correspond to the internal dissipations in the viscoelastic fluid. To evaluate the residual displacements quantitatively, the internal dissipations were calculated from all the deformations which the fluid had undergone. The reproduced line from the calculated internal dissipations coincided well to the residual displacements obtained by the experiment. In the calculation of the internal dissipation, a "Maxwell model with non-linear spring" was proposed and adopted as a model of the stretching molecules.

\section{Acknowledgments}

This work was supported by the Global COE (Center of Excellence) Program by the MEXT (Ministry of Education, Culture, Sports, Science and Technology in Japan).

\section{References}

Astarita, G., Apuzzo, G., 1965. Motion of gas bubbles in nonNewtonian liquids. AIChE J. 11, 815-820.

Arigo, M.T., McKinley, G.H., 1998. An experimental investigation of negative wakes behind spheres settling in a shear-thinning viscoelastic fluid. Rheol. Acta 37, 307-327.

Arigo, M.T., Rajagopalan, D.R., Shapley, N.T., McKinley, G.H., 1995. Sedimentation of a sphere through an elastic fluid. Part 1. Steady motion. J. Non-Newtonian Fluid Mech. 60, 225-258.

Bisgaard, C. Hassager, O., 1982. An experimental investigation of velocity fields around spheres and bubbles moving in non-Newtonian liquids. Rheol. Acta 21, 537-539.

Bird, R.B., Armstrong, R.C., Hassager, O.C., 1987. Dynamics of Polymeric Liquids 2nd ed., Wiley, New York.

Frank, X., Li, H.Z., 2006. Negative wake behind a sphere rising in viscoelastic fluids: A lattice Boltzmann investigation. Phys. Rev. E 74, 056307.

Funfschilling, D., Li, H.Z., 2001. Flow of non-Newtonian fluids around bubbles: PIV measurements and birefringence visualization. Chem. Eng. Sci. 56, 1137-1141.

Harlen, O.G., 2002. The negative wake behind a sphere sedimenting through a viscoelastic fluid. J. Non-Newtonian Fluid Mech. 108, 411-430.

Hassager, O., 1979. Negative wake behind bubbles in non-Newtonian liquids. Nature 279, 402.

Kemiha, M., Frank, X., Poncin, S., Li, H.Z., 2006. Origin of the negative wake behind a bubble rising in non-Newtonian fluids. Chem. Eng. Sci. 61, 4041-4047.

Lin, T.J., and Lin, G.M., 2005. An experimental study on flow structures of a single bubble rising in a shear-thinning viscoelastic fluid 
with a new measurement technique. Int. J. Multiphase Flow 31, 239-252.

Maalouf, A. Sigli, D., 1984. Effects of body shape and viscoelasticity on the slow flow around an obstacle. Rheol. Acta 23, 497-507.

Ohta, M., Iwasaki, E., and Obata, E., Yoshida, Y., 2003. A numerical study of the motion of spherical drop rising in shear-thinning fluid systems. J. Non-Newtonian Fluid Mech. 116, 95-111.

Satrape, J.V., Crochet, M.J., 1994. Numerical simulation of the motion of a sphere in a Bogar fluid. J. Non-Newtonian Fluid Mech. $55,91-111$.

Toms, B.A., 1949. Some observations on the flow of linear polymer solutions through straight tubes at large Reynolds numbers Proc. International Congress on Rheology 2, 135-141.

Zhang, L., Yang, C., Mao, Z.S., 2010. Numerical simulation of a bubble rising in shear-thinning fluids. J. Non-Newtonian Fluid Mech. 165, 555-567. 